\title{
A Full Digital Workflow for Computer-guided Implant Surgery in Edentulous Patients
}

Byung-Ho Choi, DDS, PhD1, Seung-Mi Jeong, DDS, PhD1, Kyoung Hee Kim, DDS ${ }^{2}$, Jing-Han Fang, DDS ${ }^{3}, X^{1}{ }^{1}{ }^{1}$ in An, DDS $^{3}$

1Professor, Department of Dentistry, Yonsei University Wonju College of Medicine, South Korea ${ }^{2}$ Resident, Department of Dentistry, Yonsei University Wonju College of Medicine, South Korea ${ }^{3}$ Graduate student, Department of Dentistry, Yonsei University Wonju College of Medicine, South Korea

\section{Background}

Computer-guided surgery has been validated since the introduction of digital technology in the early 2000s as an efficient and reliable procedure for obtaining functional and aesthetic outcomes.

\section{Objectives}

The purpose of this article is to describe a fully digital workflow that was used to perform computer-guided flapless implant placement in an edentulous patient without the use of conventional impressions, model casting and a radiographic guide.

\section{Materials and Methods}

The digital data for the workflow was acquired using an intraoral scanner and cone-beam computed tomography (СВCT). The image fusion of the intraoral scan data and the CBCT data was performed by matching the resin markers placed in the patient's mouth. The definitive digital data were then used to design a prosthetically driven implant position, a surgical template and a computer-aided design/computer-aided manufacturing (CAD/CAM)-fabricated fixed dental prosthesis. Immediate restoration was performed using surgical templates and fixed dental prosthesis immediately after implant placement. After implant placement, accuracy of implant placement was evaluated using a 3D image processing software

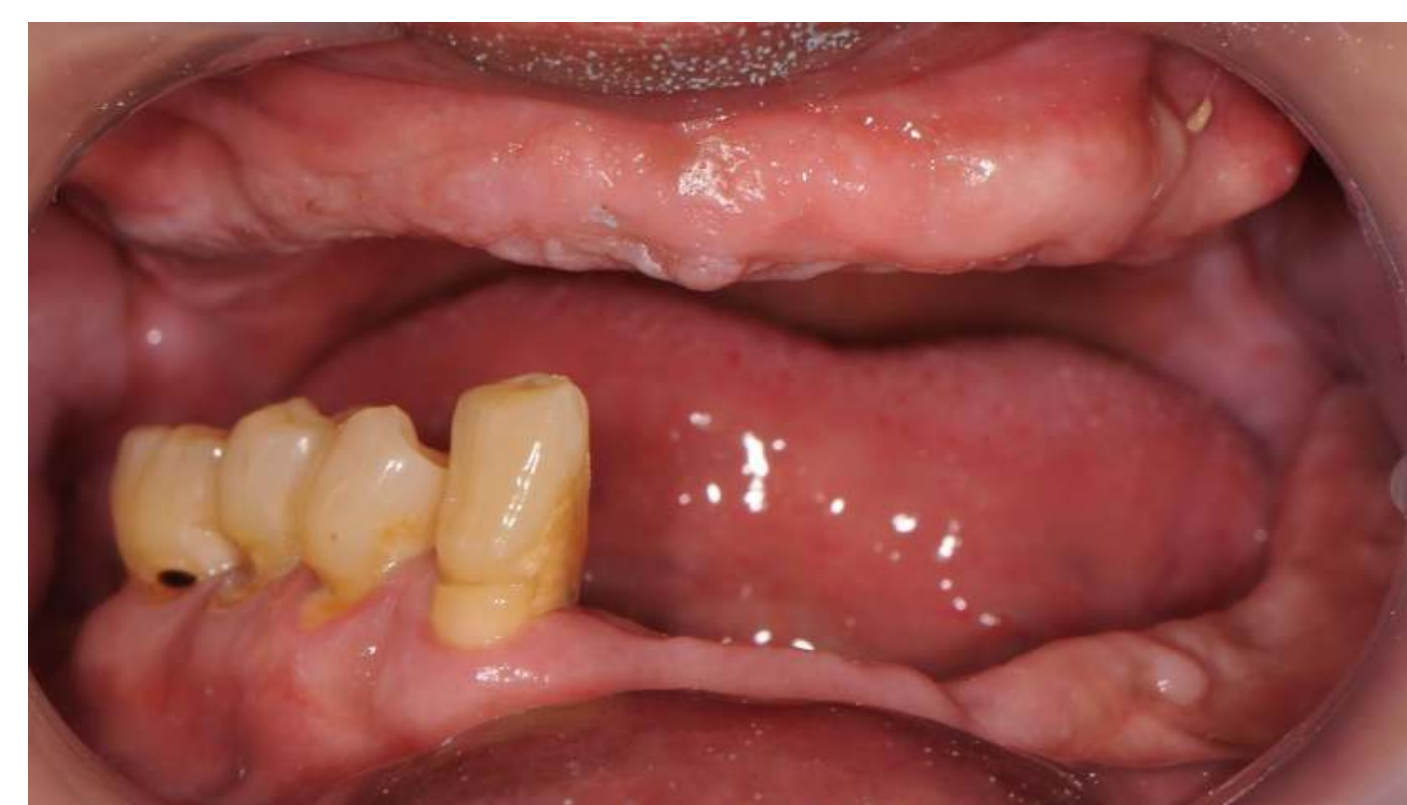

Fig.1 Intraoral photo of patient. Edentulous state on maxilla and four teeth on lower left are resident
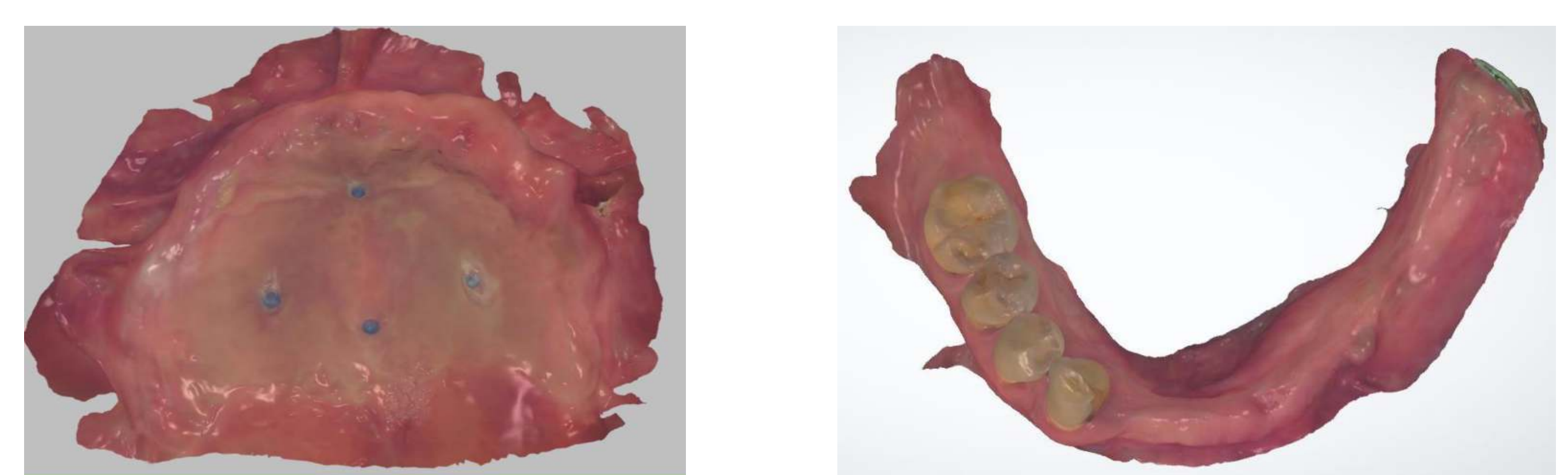

Fig.2,3 Intraoral scan image of maxilla and mandible.

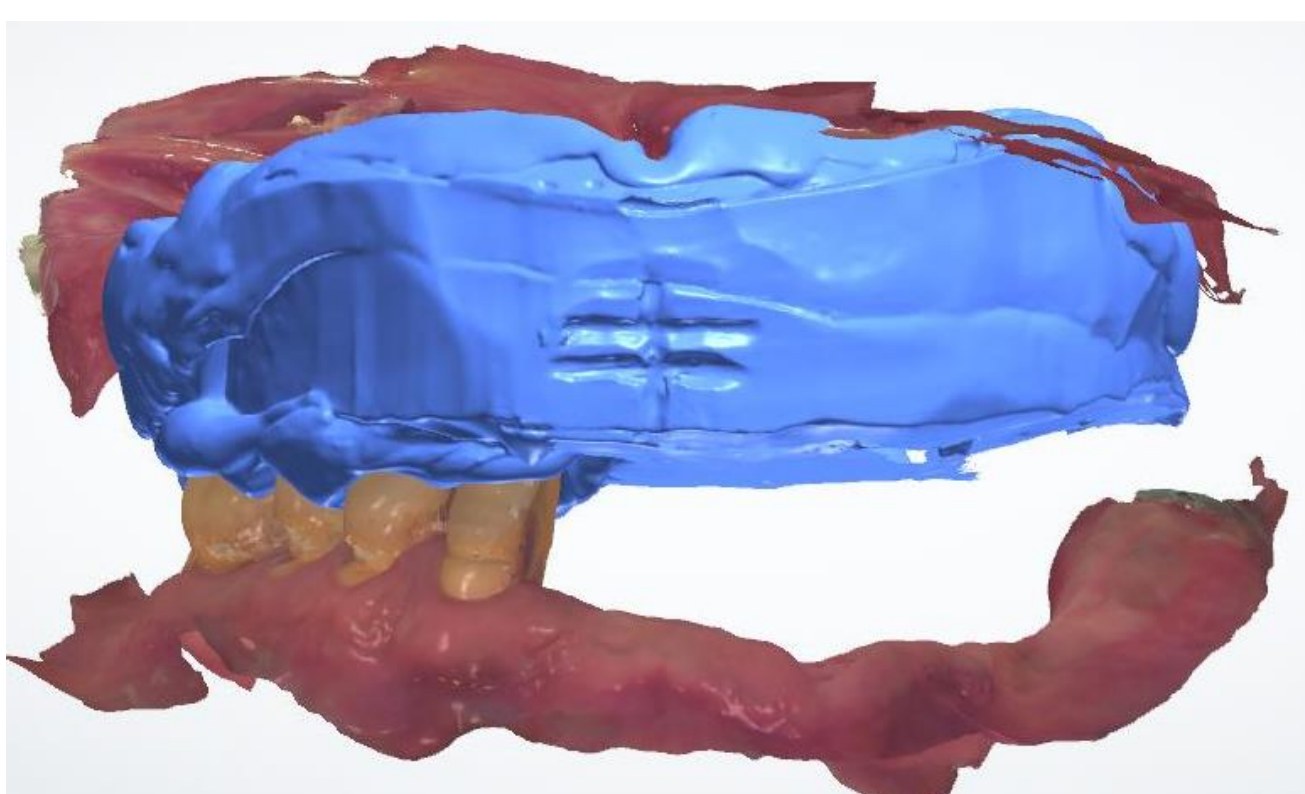

Fig.4,5 Digital scan for vertical dimension taking Using putty index, optimal vertical dimesion is taken and array The scan image of maxilla and mandible.

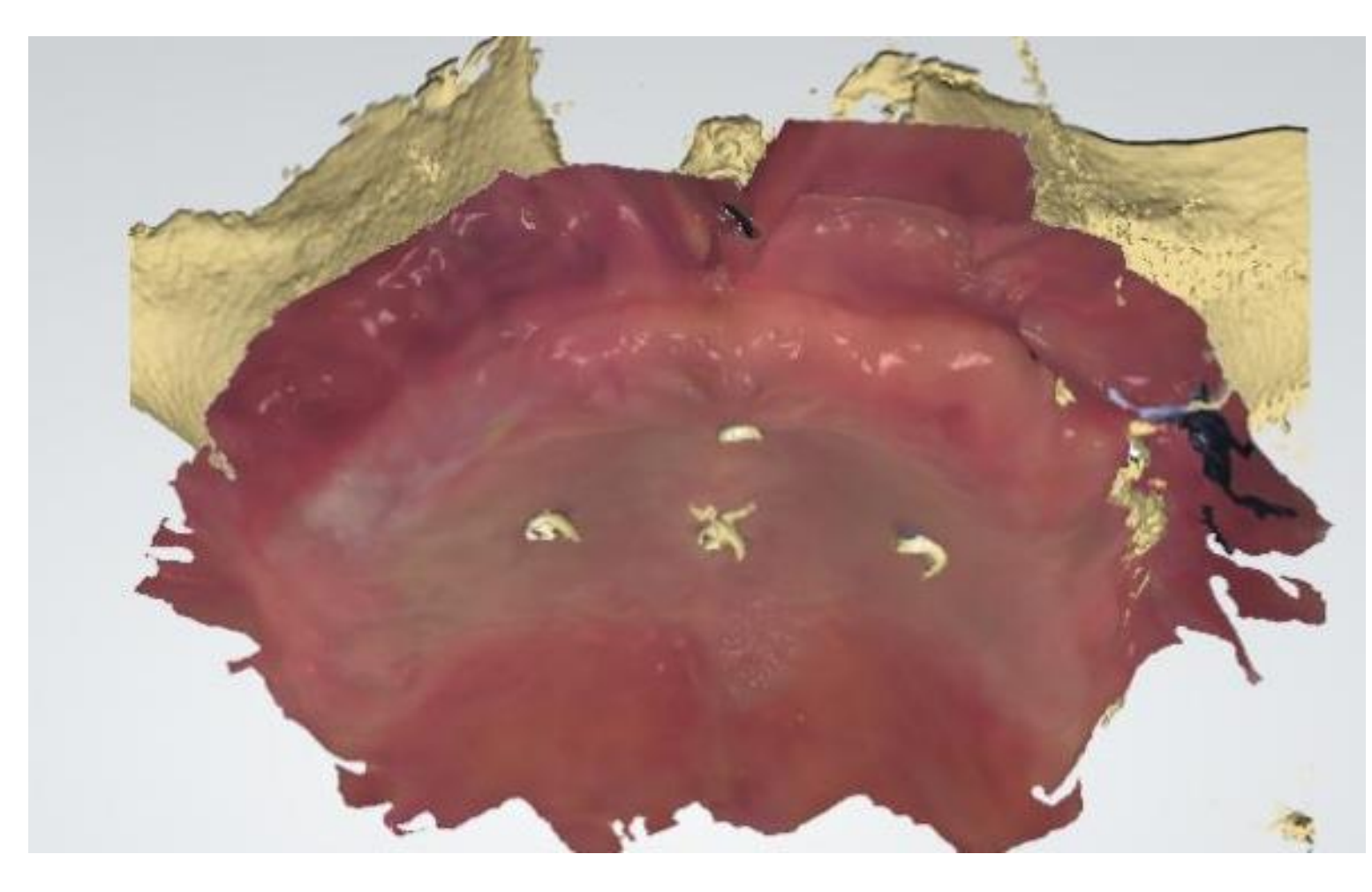

Fig.6 Fusion of data Fusion of intraoral scan data and the CBCT data by matching the resin markers place in the
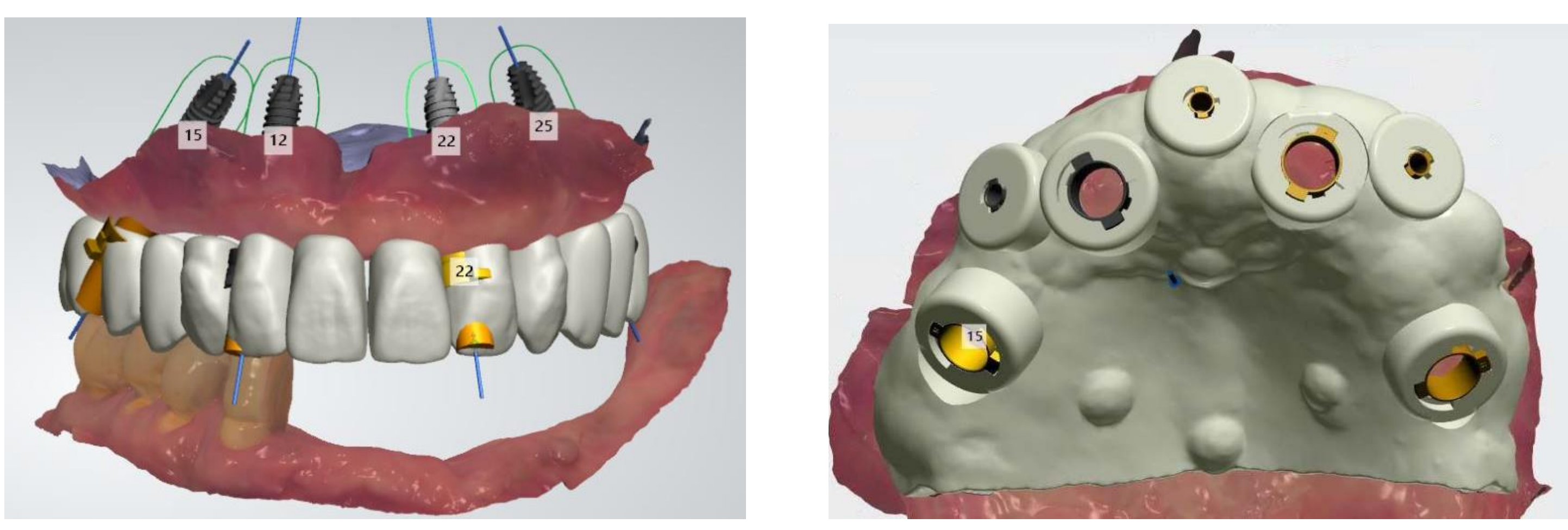

Fig.7,8 Planning of implantation Prosthetically driven implant position and a surgincal template were designed using digital data.

\section{Result}

The average angular deviation between the planned and placed implant positions was $1.1^{\circ}$ with a minimum deviation of $0^{\circ}$ and maximum deviation of $3.2^{\circ}$.
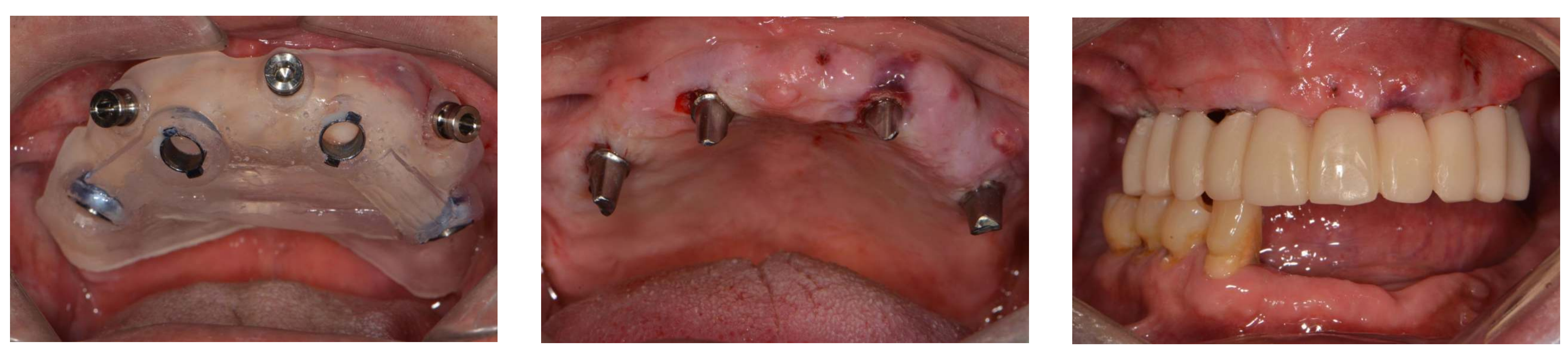

Fig.9 11 Surgical procedure and temporary prosthesis fabricated by CAD/CAM

9 Try-in and fixation of surgincal template

Fig.10 Placing of implant and abutment planned by digital devices Fig.11 Temporary restoration prefabricated by treatment planning
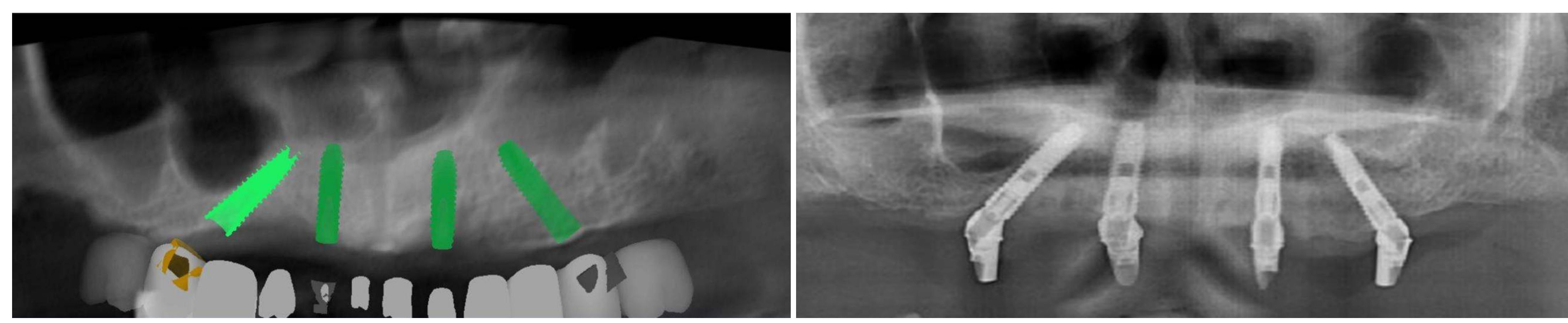

Fig.12 Panoramic image of pre-planned and placed implant It was confirmed that the planned and placed implant position are almost consistent

\section{Conclusion}

It is the digital system for implantation that simplifies the treatment process and increase accuracy. The results of this study suggest that a full digital workflow for computer-guided implant surgery in edentulous patients is a reliable method. 